\title{
Premières données sur le genre Psychomyiellodes Mosely (Trichoptera : Ecnomidae) et description d'une nouvelle espèce du Burkina Faso (Afrique Occidentale)
}

\author{
W. Guenda 1
}

Mots-clés : Psychomyiellodes, nouvelle espèce, autécologie, distribution, Afrique Occidentale, Burkina Faso.

Le matériel examiné comprend quatre espèces (Psychomyiellodes obscurus Kimmins, $P$. dentatus Kimmins, P. excavatus Kimmins et $P$. badalus n. sp.) dont une est nouvelle pour la science et deux sont signalées pour la première fois en Afrique Occidentale : $P$. dentatus et $P$. excavatus. L'étude porte sur l'autoécologie et la distribution des espèces sur une rivière de plus de mille km de long, le Mouhoun (Burkina Faso).

Preliminary data on the genus Psychomyiellodes Mosely (Trichoptera : Ecnomidae) and description of a new species in Burkina Faso (West Africa)

Keywords : Psychomyiellodes, new species, autoecology, distribution, West Africa, Burkina Faso.

Four species were identified. One of which, Psychomyiellodes badalus $\mathrm{n}$. sp. is a new species, and two $P$. dentatus and $P$. excavatus Kimmins were collected for the first time in West Africa. This study deals with the autoecology and the distribution of the species along the Mouhoun river over a distance of more than one thousand kilometers.

\section{Introduction}

En Afrique, le genre Psychomyiellodes Mosely compte sept espèces à l'heure actuelle. Elles sont toutes tropicales et décrites à partir d'échantillons de l'Afrique Centrale et de l'Est. Les différentes contributions à la connaissance de ce genre sont celles d'Ulmer (1911), Mosely (1931), Kimmins (1957, 1963), Jacquemart (1961) et Marlier (1966).

Les travaux concernant ce genre sont rares en Afrique de l'Ouest ; on peut citer Marlier (1968) qui a dressé la liste des Trichoptères du Ghana et de Côte d'Ivoire, Gibbs (1973), qui a travaillé au Ghana autour du lac Volta et Guenda $(1985,1986)$ qui a étudié le Nazinon et dressé la première liste de Trichoptères du Burkina où figure une seule espèce appartenant au genre Psychomyiellodes.

\footnotetext{
1. Université de Ouagadougou, Laboratoire d'écologie, FAST, B.P. 7021 Ouagadougou 03, Burkina Faso.
}

\section{Matériel et méthode}

La figure 1 présente la répartition géographique des 15 stations prospectées.

Les imagos ont été capturées au début de la nuit, à partir de 18 heures. Le système de capture est constitué d'une combinaison de deux lampes : une lampe à ultraviolet et l'autre à gaz.

Les individus sont disséqués et les abdomens sont éclaircis à la potasse puis montés sur lame pour examen au microscope.

\section{Résultats et discussion}

Le matériel examiné (tableau 1) comprend quatre espèces (Psychomyiellodes badalus n. sp., P. obscurus Kimmins, $P$. dentatus Kimmins et $P$. excavatus Kimmins) dont une est nouvelle pour la science et deux sont signalées pour la première fois en Afrique Occidentale : $P$. dentatus et $P$. excavatus.

Avec 229 individus récoltés, les effectifs sont relativement faibles dans l'ensemble. Seule $P$. obscurus est abondante (211 individus capturés). Elle est suivie par 


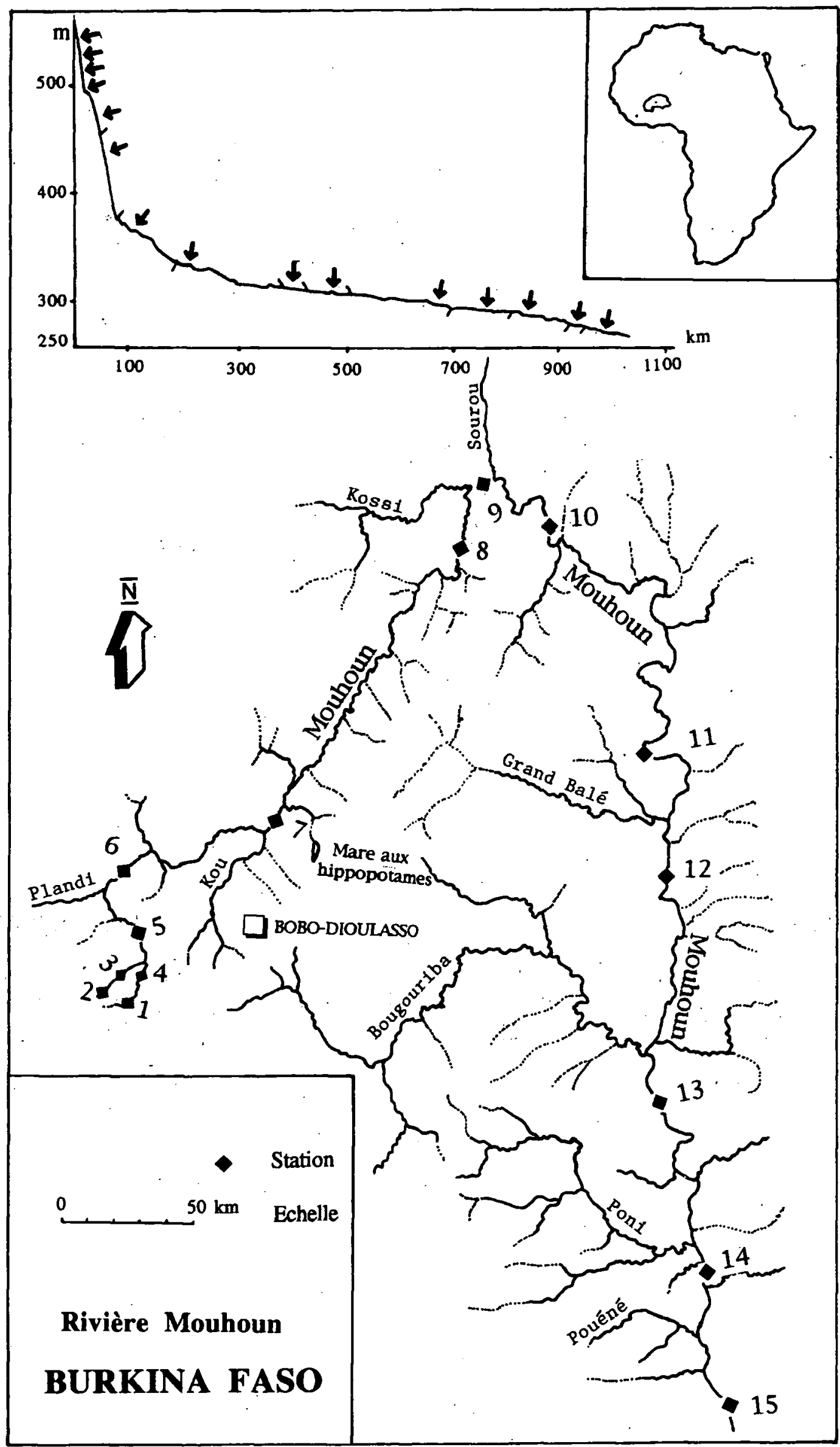

Fig. 1. Répartition des stations sur le Mouhoun : la zone des sources frontales $1:$ Tapogo $; 2:$ Orodara ; les ruisseaux alimentés par les sources 3 : Kourinion ; 4 : Fon ; le cours supérieur 5 : Badara ; 6 : Banzon ; 7 :Padéma ; le cours moyen 8 : Badala ; 9 : Kouri ; 10 : Douroula ; 11 : Boromo ; $12:$ Kayo ; le cours inférieur $13:$ Dapola ; $14:$ Zindi ; $15:$ Tantama.

Fig. 1. Location of the 15 sites in the Mouhoun river. 
Tableau 1. Effectifs des captures des adultes du genre Psychomyiellodes (Ecnomidae) à la lumière artificielle dans les stations du cours axial de la rivière Mouhoun.

Table 1. Numbers of adults of the genus Psychomyiellodes (Ecnominae) captured with artificial light in some sites of the Mouhoun river.

\begin{tabular}{|c|c|c|c|c|c|c|c|c|c|c|c|c|c|c|c|c|}
\hline $\begin{array}{c}\text { Stations } \\
\text { Espèces } \\
\end{array}$ & 1 & 2 & 3 & 4 & 5 & 6 & 7 & 8 & 9 & 10 & 11 & 12 & 13 & 12 & & 15 \\
\hline $\begin{array}{l}\text { Ecnomidae (Psychomyiellodes) } \\
\text { Psychomyiellodes obscurus }\end{array}$ & & & & 1 & & & & 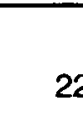 & & & 22 & & 70 & & 82 & 12 \\
\hline Psychomyiellodes dentatus & & & & & & & & 1 & & & & & & & & \\
\hline Psychomyiellodes excavatus & & & & & & & & 11 & & & 2 & & & & & \\
\hline Psychomyiellodes badalus n.sp. & & & & & & & & 3 & & & & & & & & \\
\hline
\end{tabular}

$P$. excavatus avec 14 individus, $P$. badalus n. sp. 3 individus et $P$. dentatus avec un seul individu recensé.

La systématique des adultes échantillonnés ne pose pas de problème particulier. La reconnaissance du genre est facilitée par la présence de l'éperon et les pièces génitales mâles qui répondent aux descriptions de Kimmins (1957) et de Marlier (1962) à savoir : les deux grands lobes du neuvième segment sont foliacés et dépassent les autres organes ; le pénis, semblable à celui du genre Ecnomus, est aigu et comprimé latéralement ; deux organes symétriques en forme de coupe spacieuse sont rattachés au pénis ; les gonopodes sont souvent petits, quadrangulaires et porteurs de soies internes. Cependant, Psychomyiellodes tropicus Ulmer et $P$. badalus Guenda $\mathrm{n}$. sp. font exception à la règle et présentent des gonopodes aussi développés que ceux du genre Ecnomus.

En ce qui concerne les noms d'espèces, les publications antérieures n'ont pas pris en compte l'accord masculin du genre Psychomyiellodes; en accord avec L. Botosaneanu la terminaison en $u s$ au lieu de $a$ est proposée ici : par exemple Psychomyiellodes obscurus au lieu de Psychomyiellodes obscura.

La répartition des espèces appartenant au genre $P s y$ chomyiellodes le long du Mouhoun comprend deux cas différents.

- Dans le cours moyen :

Psychomyiellodes dentatus Kimmins, P. excavatus Kimmins et $P$. badalus Guenda n. sp. y sont localisées.

- Des espèces présentes sur l'ensemble du transect et abondantes dans le cours inférieur, telle notamment $P$. obscurus Kimmins.

Ces observations montrent que la plupart des espèces sont cantonnées dans la partie aval de la rivière.
Aucune espèce ne vit dans les sources frontales et peu d'espèces colonisent le cours supérieur. Cette situation traduit bien le caractère lénitophile reconnu à la famille des Ecnomidae.

La découverte de l'espèce nouvelle pour la science montre que le genre est encore peu étudié en Afrique. L'étude des stades préimaginaux reste à faire pour toutes les espèces inventoriées.

\section{Autoécologie des espèces appartenant au genre Psychomyiellodes Mosely}

Pour chaque espèce sont désignées les stations où elle a été récoltée et les effectifs correspondants ; par exemple : St. $1(8)=$ station $^{\circ} 1$ (8 individus récoltés).

\section{- Psychomyiellodes badalus Guenda n. sp.}

St. 8 (3)

Matériel : Holotype et 2 paratypes mâles capturés le 3-11-1991 à Badala dans le cours moyen du Mouhoun près de la ville Dédougou au sud-ouest du Burkina Faso. Le matériel est actuellement placé dans la collection de l'auteur ; des paratypes seront déposés au Muséum d'Histoire Naturelle de Paris.

Morphologie générale

Cette espèce a l'allure d'un Ecnomus, de couleur sombre. La formule calcarienne est 2/4/4 et l'éperon apical interne du tibia postérieur se termine par un crochet en spirale.

Description des genitalia (Fig. 2)

Les genitalia mâles présentent l'aspect classique du genre Ecnomus en vue ventrale et dorsale. Seule la position latérale met en évidence l'organe en forme de coupe caractéristique du genre Psychomyiellodes. 


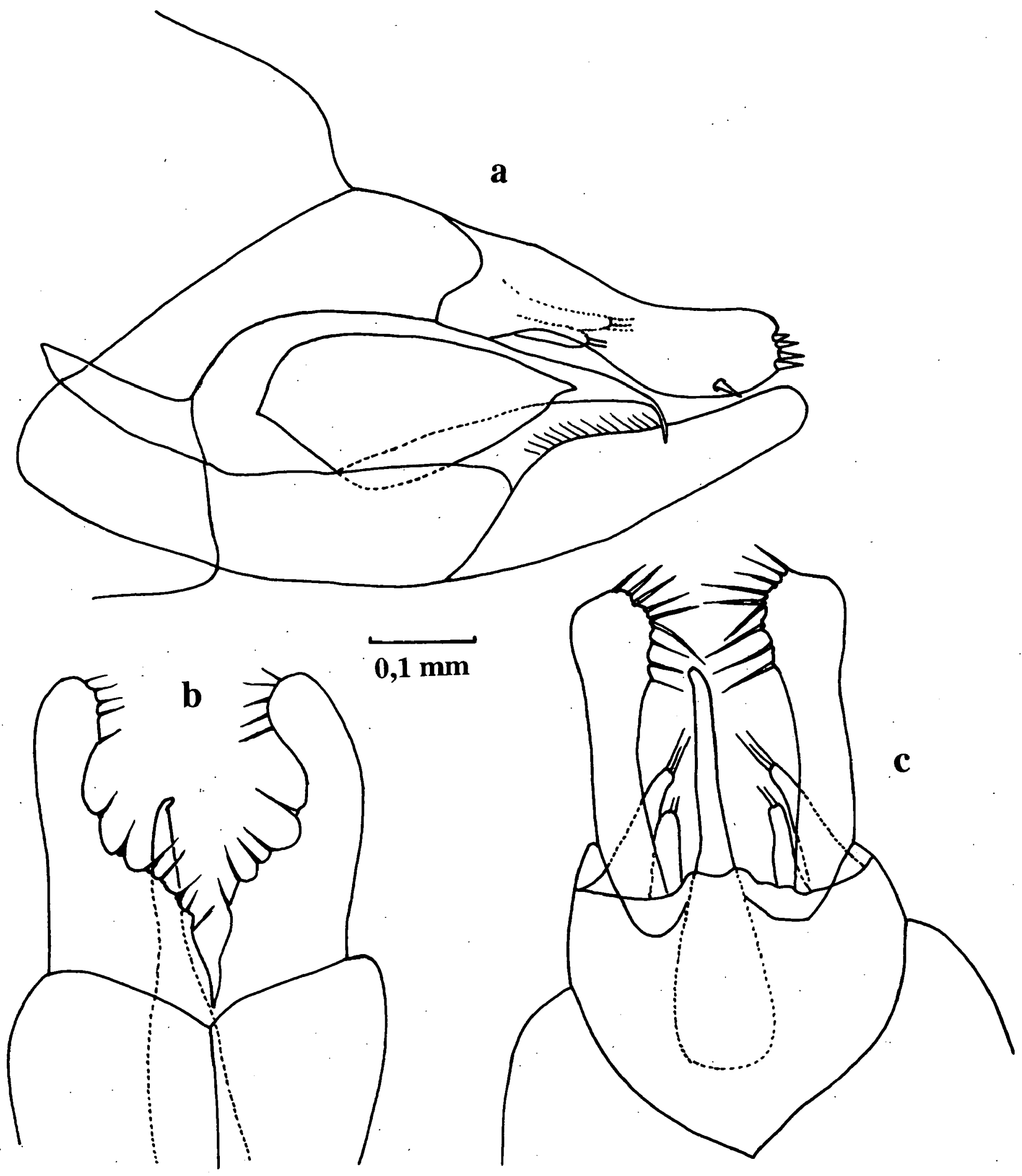

Fig. 2. Genitalia mâles de Psychomyiellodes badalus n. sp. a.: vue latérale, b : vue ventrale, c : vue dorsale. Fig. 2. Psychomyiellodes baladus $\mathrm{n}$. sp. male genitalia a : lateral, $\mathrm{b}$ : ventral, $\mathrm{c}$ : dorsal. 
Les processus du neuvième segment sont très allongés et se terminent par de nombreuses épines. A la base de ces pièces se trouvent deux processus courts terminés par deux soies courtes pour le processus proximal et par trois longues soies au niveau du processus distal.

Le neuvième sternite, très allongé, atteint le niveau du processus tergal du même segment ; ce caractère rapproche cette espèce de Psychomyiellodes tropicus. Elle en diffère par tous les autres caractères. Les pièces latérales en forme de coupe, logées dans le huitième segment, sont légèrement comprimées vers la base et terminées par des denticulations et des replis.

Le phallus, large à la base, s'amincit et se courbe progressivement pour terminer en pointe fortement recourbée.

Etymologie : Badala, un village de la région de Dédougou au Burkina Faso

\section{- Psychomyiellodes obscurus Kimmins 1957}

St. 4 (1) ; St. 8 (22) ; St. 9 (2) ; St. 11 (22) ; St. 13 (70) ; St. 14 (82) ; St. 15 (12).

C'est ici l'espèce la plus abondante du genre Psychomyiellodes. Absente du cours supérieur, cette espèce est présente à la source et colonise les cours moyen et inférieur du Mouhoun. Son absence du cours supérieur pourrait être due à la faible couverture végétale des berges de cette zone.

Elle est déjà signalée au Burkina (Guenda 1985, 1986) sur la rivière Nazinon. Décrite de l'Uganda, elle est connue au Zaïre (Marlier 1958).

Sa distribution pourrait comprendre les cours stabilisés des grandes rivières.

La biologie de Psychomyiellodes obscurus n'est pas encore connue.

\section{- Psychomyiellodes dentatus Kimmins 1957}

\section{St. 8 (1).}

Espèce rare, elle a été capturée à Badala dans le cours moyen. Elle est sténotope exclusive du cours moyen.

Décrite du lac Victoria (Kimmins 1957) elle n'avait pas encore été signalée en Afrique de l'Ouest. Sa biologie est inconnue. Elle pourrait avoir une large distribution dans la région éthiopienne.

\section{- Psychomyiellodes excavatus Kimmins 1963}

St. 8 (11) ; St. 9 (1) ; St. 10 (2).

Sans être abondante, cette espèce est cantonnée dans le cours moyen du Mouhoun ; c'est son milieu électif.

Décrite de l'Afrique de l'Est, elle n'était pas connue en Afrique Occidentale.

Sa biologie est inconnue. Elle pourrait être présente dans les grandes rivières de l'Afrique tropicale.

\section{Remerciements}

Je remercie le Bureau de Coopération internationale de l'Université de Groningen au Pays-Bas pour son soutien matériel dans la réalisation de ce travail ; mes remerciements vont également à Monsieur L. Botosaneanu pour l'examen d'une partie des échantillons et pour ses précieux conseils.

\section{Références}

Gibbs D.G. 1973. - The Trichoptera of Ghana. Dt. ent. Z., 20, 4-5 : 363-424.

Guenda W. 1985. - Hydrobiologie d'un cours d'eau temporaire en zone soudanienne : la Volta Rouge (Burkina Faso - Ghana). Relation avec les traitements chimiques antisimulidiens. Thèse de $3^{e}$ cycle. Univ. Aix-Marseille : 193 p.

Guenda W. 1986-1987. - Evaluation de la toxicité de l'Abate sur les macroinvertébrés de la Volta Rouge (Burkina Faso - Ghana). Bulletin de l'IFAN, T.46, sér. A, nº 3-4 : 333-349.

Jacquemart S. 1961. - Trichoptera. Exploration du Parc National de l'Upemba (Mission de G.F. De Witte) Inst. parcs. Nat. Congo et Rwanda-Burundi. $62: 3-45$.

Kimmins D.E. 1957. - Notes on the Psychomyidae (Trichoptera) from the African mainland (south of the Mediterranean region) with particular reference to the genera Ecnomus and Psychomyiellodes. Trans. R. ent. Soc. Lond., 109, 8 : 259-273.

Kimmins D.E. 1963. - On the Trichoptera of Ethiopia. Bull. Brit. Mus. (Nat. Hist.) Ent., 13, $5: 119-170$.

Marlier G. 1958. - Trichoptères du lac Tumba. Extr. Bull. Ann. Soc. Roy. Entom. Belg., 94, 11-12:301-320.

Marlier G. 1962. - Genera des Trichoptères de l'Afrique. Ann. Mus. Roy. Afr. Centr., Sér. 8, Zool. 109 : 7-259.

Marlier G. 1966. - Les Trichoptères du Musée de Dundo. Comp. Diam. Angol. Mus. Dundo, $30: 17-79$.

Marlier G. \& Botosaneanu L. 1968. - Trichoptères du Ghana et de la Côte d'Ivoire. Bull. Inst. Roy. Sc. Nat. Belg., 44, 16:16p.

Mosely M.E. 1932. - Contribution à l'étude de la faune du Mozambique. Voyage de M.P. Lesne (1928-1929). 12 : Trichoptera. Mém. est ud. Mus. Zool. Univ. Coimbra, 1, 64 : 1-5.

Ulmer G. 1912. — Trichopteren von Acquatorial Afrika. Wiss. Ergeb. Deutsch. Zentr. Apr. Exp., 1907-1908. 4, 2 : 81-125. 